\title{
A. LEMMA WHICH DISTINGUISHES MINIMAL LOGICS FROM OTHER LOGICS
}

\author{
KATUZI ONO
}

In my joint work with J. Ito [7], we have pointed out that the following property (called ASSUMPTION REMOVABILITY) is characteristic of positive logics LO, LP, and $\mathbf{L} \mathbf{Q}^{1)}$ :

Assumption Removability. If $\mathfrak{A} \rightarrow \mathfrak{B}$ is provable for any pair of propositions $\mathfrak{U}$ and $\mathfrak{B}$ having no primitive notions (proposition-, predicate-, and relation-symbols) in common, then $\mathfrak{B}$ is also provable.

ASSUMPTION REMOVABILITY characterizes these positive logics, since it holds for them but for none of the lower classical predicate logic LK, the intuitionistic predicate logic $\mathbf{L} \mathbf{J}$, and the minimal logics LM (intuitionistic) and LN (classical) ${ }^{2}$. In the present paper, I will further point out that LM and $\mathbf{L N}$ can be distinguished from $\mathbf{L J}, \mathbf{L K}, \mathbf{L P}$, and $\mathbf{L Q}$ by the following lemma (called SEPARABILITY LEMMA in the present paper):

Separability Lemma. If $\mathfrak{\mathfrak { U }}_{1} \wedge \mathfrak{B}_{1} \rightarrow \mathfrak{Q}_{2} \vee \mathfrak{B}_{2}$ is provable and $\mathfrak{P}_{1} \rightarrow \mathfrak{A}_{2}$ and $\mathfrak{B}_{1} \rightarrow \mathfrak{B}_{2}$ have no primitive notions in common, then either $\mathfrak{A}_{1} \rightarrow \mathfrak{U}_{2}$ or $\mathfrak{B}_{1} \rightarrow \mathfrak{B}_{2}$ is provavle.

Naturally, LJ and LK are formulated in Gentzen's manner as Gentzen's LJ and $\mathbf{L K}^{3)}$, respectively. $\mathbf{L P}$ and $\mathbf{L Q}$ can be also formulated in Gentzen's manner as the sub-logics of $\mathbf{L J}$ and $\mathbf{L K}$ having the logical constants $\rightarrow, \wedge, \vee,()$, and $(\exists)$, respectively. It should be also remarked here that Gentzen's cutelimination theorem ${ }^{4}$ holds for all the logics $\mathbf{L J}, \mathbf{L K}, \mathbf{L P}$, and LQ formulated in Gentzen's manner.

By the following two theorems, I show that we can distinguish LM and LN

Received April 2, 1966.

1) As for the primitive logic LO, see Ono [6]. As for the intuitionistic positive logic LP and the classical positive logic LQ, see Curry [2], Lorenzen [5], and Ono [6]. Curry refers to $\mathbf{L P}$ and $\mathbf{L Q}$ by $\mathbf{L A}$ and $\mathbf{L C}$, respectively. LE.

2) Johansson introduced LM (Minimalkalkül) in [4]. In [2], Curry refers to LN by

3) As to Gentzen's LJ and LK, see Gentzen [3].

4) The HAUPTSATZ of Gentzen [3]. 
from LJ, LK, LP, and LQ by SEPARABILITY LEMMA.

TheOREM 1. SEPARABILITY LEMMA holds for none of LM and $\mathbf{L N}$.

Proof. I will prove this theorem by giving the following counter example:

$$
\rightarrow(A \rightarrow A) \wedge B \cdot \rightarrow \cdot A \vee \rightarrow B
$$

is surely provable in $\mathbf{L M}$ as well as in $\mathbf{L N}$. On the other hand, none of

$$
\neg(A \rightarrow A) \rightarrow A \text { and } B \rightarrow \neg B
$$

is provable in any of one these logics, although these two propositions have no primitive notions in common.

THEOREM 2. SEPARABILITY LEMMA holds for any one of $\mathbf{L J}, \mathbf{L K}, \mathbf{L P}$, and $\mathbf{L Q}$.

Proof. Let $\mathbf{L}$ be any one of the logics $\mathbf{L J}, \mathbf{L K}, \mathbf{L P}$, and $\mathbf{L Q}$ formulated in Gentzen's manner, and let $\mathfrak{P}_{1} \wedge \mathfrak{B}_{1} \rightarrow \mathfrak{U}_{2} \vee \mathfrak{B}_{2}$ be any proposition provable in $\mathbf{L}$ where $\mathfrak{A}_{1} \rightarrow \mathfrak{U}_{2}$ and $\mathfrak{B}_{1} \rightarrow \mathfrak{B}_{2}$ have no primitive notions in common. Then, the sequent $\mathfrak{A}_{1}, \mathfrak{B}_{1} \mid-\mathfrak{A}_{2} \vee \mathfrak{B}_{2}{ }^{5)}$ must be provable in $\mathbf{L}$. Accordingly, by the cutelimination theorem, we can assume that the same sequent can be proved by a proof $I$ in $\mathbf{L}$ by making use of no cuts.

For any sequent $\Gamma \mid-\Delta$ in $\Pi$, new sequents $\Gamma_{a} \mid-\Delta_{a}$ and $\Gamma_{b} \mid-\Delta_{b}$ are defined by the following:

$\Gamma_{a}$ (or $\Gamma_{b}$ ) is the sequence of all the propositions in $\Gamma$ which have at least one primitive notion in common with $\mathfrak{A}_{1} \rightarrow \mathfrak{A}_{2}$ (or with $\mathfrak{B}_{1} \rightarrow \mathfrak{B}_{2}$ ). $\Delta_{a}$ as well as $\Delta_{b}$ is defined similarly.

Any sub-formula of $\mathfrak{A}_{1}$ and $\mathfrak{A}_{2}$ (or $\mathfrak{B}_{1}$ and $\mathfrak{B}_{2}$ ) is called an $a$-formula (a $b$-formula). It is remarkable that, for any sequent $\Gamma \mid-\Delta$ in $\Pi$, any proposition in $\Gamma_{a}$ (or in $\Gamma_{b}$ ) is an $a$-formula (a $b$-formula) and any proposition in $\Delta_{a}$ (or in $\Delta_{b}$ ) is either an $a$-formula (a $b$-formula) or the proposition $\mathfrak{A}_{2} \vee \mathfrak{B}_{2}$.

Further, we call any sequent $\Gamma \mid-\Delta$ in $\Pi$ an $a$-sequent (or a $b$-sequent) if and only if $\Gamma_{a} \mid-\Delta_{a}$ (or $\Gamma_{b} \mid-\Delta_{b}$ ) is provable in $\mathbf{L}$.

Evidently, any fundamental sequent of $\Pi$ is an $a$-sequent or a $b$-sequent, and any sequent deduced from an $a$-sequent or a pair of $a$-sequents (a $b$-sequent

5) I employ the notation $\Gamma \mid-\Delta$ in place of Gentzen's original notation $\Gamma \rightarrow \Delta$, because I denote IMPLICATION by $\rightarrow$ (Gentzen denoted it by $\supset$ ). As for inference rules of $\mathbf{L J}$ and $L K$, see Gentzen [3]. 
or a pair of $b$-sequents) is an $a$-sequent (a $b$-sequent).

Moreover, we can confirm that any sequent in $I I$ deduced from a pair of an $a$-sequent and a $b$-sequent is either an $a$-sequent or a $b$-sequent. To show this, we have only to check the following three types of inferences:

$$
\begin{aligned}
& \text { Type I : } \frac{\Gamma|-\Delta, \mathfrak{F} \Gamma|-\Delta, \mathbb{B},}{\Gamma \mid-\Delta, \tilde{F} \wedge(\mathbb{B}} \\
& \text { Type II : } \quad \frac{\Gamma, \mathfrak{F}|-\Delta I, \mathbb{B}|-\Delta}{\Gamma, \mathfrak{f} \vee \mathbb{B} \mid-\Delta} \text {, } \\
& \text { Type III : } \quad \frac{\Gamma \mid-\Delta, \tilde{v}}{\Gamma, \tilde{\mho} \rightarrow|\mathbb{( \$ 1}|-\Delta, \Lambda} \text {. }
\end{aligned}
$$

Let us now check any inference of Type I (Type II ; Type III) which really occurs in $I$, assuming that one of its over-sequents $\Gamma \mid-\Delta, \mathfrak{\mho}$ and $\Gamma \mid-\Delta, \&(\Gamma$, $\tilde{F} \mid-\Delta$ and $\Gamma, \mathbb{S}|-\Delta ; \Gamma|-\Delta, \tilde{F}$ and $\Gamma, \mathbb{S} \mid-\Lambda)$ is an $a$-sequent and the other is a $b$-sequent. It should be noticed that $\mathfrak{F} \wedge \mathbb{B}(\mathfrak{F} \vee \mathbb{S} ; \widetilde{F} \rightarrow \mathbb{B})$ of the inference is either an $a$-formula or a $b$-formula.

Case I: $\tilde{F} \wedge\left(\mathbb{S}(\mathfrak{F} \vee \mathbb{S} ; \mathfrak{F} \rightarrow \mathbb{B})\right.$ be an $a$-formula. $\Gamma_{b} \mid-\Delta_{b}\left(I_{b} \mid-\Delta_{b}\right.$; either $\Gamma_{b} \mid-\Delta_{j}$ or $\Gamma_{b} \mid-\Lambda_{b}$ ) must be provable in $\mathbf{L}$, because one of the over-sequents of the inference is assumed to be a $b$-sequent. Accordingly, $\Gamma_{b} \mid-\left(\Delta, \mho \wedge(B)_{b}\right.$ i.e. $\Gamma_{b} \mid-\Delta_{b}\left(\left(I, \mathfrak{j} \vee \vee(S)_{b} \mid-\Delta_{b}\right.\right.$ i.e. $\Gamma_{b}\left|-\Delta_{b} ;(\Gamma, \tilde{F} \rightarrow(B))_{b}\right|-(\Delta, \Lambda)_{b}$ i.e. $\left.\Gamma_{b} \mid-\Delta_{b}, \Lambda_{b}\right)$ must be also provable in $\mathbf{L}$. Hence, the under-sequent $\Gamma \mid-\Delta, \mathfrak{F} \wedge \dot{S}(\Gamma, \mathfrak{F} \vee \& \mid-\Delta$; $\Gamma, \mathfrak{F} \rightarrow(\mathbb{B} \mid-\Delta, A)$ of the inference is a $b$-sequent.

Case II : $\mathfrak{F} \wedge \mathbb{B}(\mathfrak{F} \vee \mathbb{B} ; \mathfrak{F} \rightarrow \mathbb{B})$ be a $b$-formula. In this case, the undersequent of the inference is proved to be an $a$-sequent quite similarly as in Case I.

Consequently, $\mathfrak{A}_{1}, \mathfrak{B}_{1} \mid-\mathfrak{A}_{2} \vee \mathfrak{B}_{2}$ must be an $a$-sequent or a $b$-sequent.

Now, let $\mathfrak{N}_{1}, \mathfrak{B}_{1} !-\mathfrak{A}_{2} \vee \mathfrak{B}_{2}$ be an $a$-sequent. Then, $\mathfrak{A}_{1} \mid-\mathfrak{A}_{2} \vee \mathfrak{B}_{2}$ must be provable by a proof $\Pi_{a}$ by making use of no cuts. Any proposition occurring in $\Pi_{a}$ must be either the proposition $\mathfrak{H}_{2} \vee \mathfrak{B}_{2}$ or a sub-formula of $\mathfrak{P}_{1}$ or $\mathfrak{A}_{2}$. Now, the proof figure obtained on replacing every proposition $\mathfrak{A}_{2} \vee \mathfrak{B}_{2}$ occurring in $\Pi_{a}$ by $\mathfrak{H}_{2}$ is proved to be reducible to a right proof figure of $\mathfrak{A}_{1} \mid-\mathfrak{N}_{2}$ in $\mathbf{L}$. Hence, $\mathfrak{P}_{1} \rightarrow \mathfrak{A}_{2}$ must be provable in $\mathbf{L}$.

We can show similarly that $\mathfrak{B}_{1} \rightarrow \mathfrak{B}_{2}$ is provable in $\mathbf{L}$ in the case where $\mathfrak{U}_{1} \wedge \mathfrak{B}_{1} \mid-\mathfrak{U}_{2} \vee \mathfrak{B}_{2}$ is a $b$-sequent. 
Remark 1. If SEPARABILITY LEMMA is assumed in LM, the logic turns out to be LJ. For, SEPARABILITY LEMMA in LM gives rise to the rule that any contradictory proposition implies every proposition. Namely, let $\mathfrak{A}$ be any contradictory proposition, 5 be any proposition, and $B$ be any proposition symbol which occurs neither in $\mathfrak{A}$ nor in 5 . Because $\mathfrak{A} \rightarrow \neg B$ is provable in $\mathbf{L M}, \mathfrak{U} \wedge(B \rightarrow B) \cdot \rightarrow \cdot(5 \vee \neg B$ is also provable in $\mathbf{L M}$. If we assume SEPARABILITY LEMMA in LM, either $\mathfrak{U} \rightarrow \circlearrowleft$ or $(B \rightarrow B) \rightarrow \rightarrow B$ i.e. $\rightarrow B$ must be provable in LM. However, $\neg B$ is not provable for any proposition symbol $B$, so $\mathfrak{A} \rightarrow \circlearrowleft$ must be provable in LM. Accordingly, any contradictory proposition $\mathfrak{A}$ implies every proposition (5 as far as SEPARABILITY LEMMA is assumed in LM. This seems to justify to adopt the occasionally debatable inference rule that contradiction implies everything.

If we assume SEPARABILITY LEMMA in any logic stronger than LM, we have a logic stronger than $\mathbf{L J}$, an intermediate logic (a logic lying between $\mathbf{L J}$ and LK, see Umezawa [9]) in general. However, it is still an open question for me whether SEPARABILITY LEMMA holds for intermediate logics in general or not.

Remark 2. For the lower classical predicate logic LK, SEPARABILITY LEMMA is a consequence of Craig's interpolation theorem (See Craig [1]). Namely, in $\mathbf{L K}$, the proposition $\mathfrak{A}_{1} \wedge \mathfrak{B}_{1} \rightarrow \mathfrak{A}_{2} \vee \mathfrak{B}_{2}$ is equivalent to

$$
\rightarrow\left(\mathfrak{U}_{1} \rightarrow \mathfrak{U}_{2}\right) \rightarrow\left(\mathfrak{V}_{1} \rightarrow \mathfrak{B}_{2}\right) .
$$

If we assume that this proposition is provable in LK for propositions $\mathfrak{A}_{1} \rightarrow \mathfrak{U}_{2}$ and $\mathfrak{B}_{1} \rightarrow \mathfrak{B}_{2}$ having no primitive notions in common, then either $\rightarrow \rightarrow\left(\mathfrak{A}_{1} \rightarrow \mathfrak{A}_{2}\right)$ i.e. $\mathfrak{P}_{1} \rightarrow \mathfrak{P}_{2}$ or $\mathfrak{B}_{1} \rightarrow \mathfrak{B}_{2}$ must be provable according to Craig's interpolation theorem.

Interpolation theorem is also proved for intuitionistic predicate logic LJ by Schütte (See Schütte [8]). With respect to logics other than LK, however, I can only say the following: The special case of the interpolation theorem for LJ, where assumption and consequent have no primitive notion in common, follows immediately from the SEPARABILITY LEMMA. The ASSUMPTION REMOVABILITY asserts something more for the special case of the interpolation theorem with respect to positive logics. 


\section{REFERENCES}

[1] Craig, W.: Linear reasoning. A new form of the Herbrand-Gentzen theorem. J. Symb. Log., 22 (1957), 250-268.

[2] Curry, H. B.: Foundations of mathematical logic. New-York (1963).

[3] Gentzen, G.: Untersuchungen über das logische Schliessen. Math. Z., 39 (1934), 176$210,405-431$.

[4] Johansson, I.: Der Minimalkalkül, ein reduzierter intuitionistischen Formalismus. Compositio Math., 4 (1936), 119-136.

[5] Lorenzen, P.: Einführung in die operative Logik und Mathematik. Berlin-GöttingenHeidelberg, (1955).

[6] Ono, K.: On universal character of the primitive logic. Nagoya Math. J., 27-I (1966), 331-353.

[7] Ono, K. and Ito, J.: On a charcteristic feature of the positive logics. Nagoya Math. J., 28 (1966), 193-196.

[8] Schütte, K.: Der Interpolationssatz der intuitionistischen Prädikatenlogik, Math. Ann., 148 (1962), 192-200.

[9] Umezawa, T.: On logics intermediate between intuitionistic and classical predicate logic. J. Symb. Log., 24 (1959), 141-153.

Mathematical Institute,

Nagoya University 